Selection of reference genes for gene expression studies in rat oligodendrocytes using quantitative real time PCR

Non Peer-reviewed author version

NELISSEN, Katherine; SMEETS, Karen; Mulder, M.; HENDRIKS, Jerome \& AMELOOT, Marcel (2010) Selection of reference genes for gene expression studies in rat oligodendrocytes using quantitative real time PCR. In: JOURNAL OF NEUROSCIENCE METHODS, 187. p. 78-93.

DOI: $10.1016 /$ j.jneumeth.2009.12.018

Handle: http://hdl.handle.net/1942/10854 


\title{
Selection of reference genes for gene expression studies in rat oligodendrocytes using quantitative real time PCR
}

\author{
Katherine Nelissen ${ }^{1}$, Karen Smeets ${ }^{2}$, Monique Mulder ${ }^{3}$, Jerome J.A. Hendriks ${ }^{1}$, Marcel \\ Ameloot $^{1}$
}

${ }^{1}$ Biomedical Research Institute, Hasselt University and transnational University Limburg, Agoralaan Gebouw C, B-3590 Diepenbeek, Belgium

${ }^{2}$ Centre for Environmental Sciences, Hasselt University, Agoralaan Gebouw D, B-3590

Diepenbeek, Belgium

${ }^{3}$ Department of Internal Medicine, Division of Pharmacology, Vascular and Metabolic

Diseases, Rotterdam University,'s Gravendijkwal 230, 3015 CE Rotterdam, The Netherlands

Number of text pages: 25

Number of figures: 4

Number of tables: 3

Contact information corresponding author:

Prof. Dr. Marcel Ameloot

Hasselt University

Laboratory of cellphysiology

Biomedical Research Institute

Agoralaan Building C

B-3590 Diepenbeek

BELGIUM

Tel: +3211269233

Fax: +32 11269299

marcel.ameloot@uhasselt.be 


\section{Abstract}

Quantitative real time polymerase chain reaction (qPCR) has become a widely used tool to examine gene expression levels. Reliable quantification, however, depends on a proper normalization strategy. Normalization with multiple reference genes is becoming the standard, although the most suitable reference genes depend on the applied treatment as well as the tissue or cell type studied. In this study the stability of various reference genes was investigated in cultures of oligodendrocytes derived from either mature or neonatal rats, the latter also in the presence of the liver X receptor (LXR) agonist.

The expression stability of ten commonly used reference genes (HPRT, GAPDH, 18S, ActB, CycA, Tbp, Rpl13A, YWHAZ, HMBS, Pgk1) was analyzed using geNorm and NormFinder. When comparing the different types of cell cultures, Rpl13A, CycA, Pgk1 and YWHAZ were identified as most stable genes. After LXR agonist treatment, CycA, Pgk1 and Rpl13A were found to be the most stable by both geNorm and NormFinder. HMBS and the commonly used housekeeping genes GAPDH and $18 \mathrm{~S}$ turned out to be the most variable according to geNorm and NormFinder. In conclusion, the use of multiple reference genes, instead of only one, in qPCR experiments with rat oligodendrocytes is strongly advised and standard housekeeping genes such as GAPDH and $18 \mathrm{~S}$ are not recommended as they appear to be relatively unstable under the experimental conditions used. Reference gene selection should always be performed for each individual experiment, since useful reference genes are very specific for every situation.

Keywords glial cells, internal control gene, normalization, geNorm, NormFinder 


\section{Introduction}

Oligodendrocytes (OLGs) are the myelin-forming glial cells of the central nervous system (CNS). The myelin sheath is a fatty insulating layer that surrounds axons of neurons. Over $25 \%$ of the lipid content in myelin consists of cholesterol. Mice that lack the ability to produce cholesterol in their OLGs show impaired myelination (Saher et al., 2005). Another indication of the importance of the cholesterol metabolism in myelinating OLG was found in liver X receptor (LXR) knockout mice. LXRs are oxysterol activated nuclear receptors, which play an important part in the control of cellular and whole-body cholesterol homeostasis (Peet et al., 1998; Repa and Mangelsdorf, 2000). Absence of LXRs in mice leads to disorganized myelin sheaths, which indicates a role for the LXRs in OLG function (Wang et al., 2002). Although OLGs are important cholesterol producing cells in the CNS, most of the research on the CNS cholesterol homeostasis has focused on neurons and astrocytes. We investigate the role of OLGs in CNS cholesterol trafficking by studying the effect of LXR agonist (Schultz et al., 2000) treatment on expression levels of cholesterol related genes.

For measuring molecular responses, $\mathrm{qPCR}$ is the most sensitive and reliable method to detect and quantify specific mRNA expression levels. Proper use of this method requires normalization to account for the differences in amount of starting material, variability in RNA quality, variable PCR or cDNA synthesis efficiencies and differences between tissues and cell types in overall transcriptional activity (Vandesompele et al., 2002). The most frequently applied normalization method is the use of internal reference genes. Until recently, "housekeeping genes" (HKGs) were commonly used as reference genes. HKGs encode proteins that provide basic, essential functions that all cells need to survive. They are supposed to have stable expression levels in different cell types and tissues, across developmental stages and various conditions. Several studies, however, have demonstrated 
that expression levels of traditional HKGs can vary under different experimental conditions (Deindl et al., 2002; Dheda et al., 2005; Radonic et al., 2004). In addition, normalization to a single control gene can lead to erroneous normalization. The use of at least three reference genes for the correct normalization of qPCR data has been proposed (Vandesompele et al., 2002).

The search for suitable reference genes was complicated by the fact that for the evaluation of the expression stability of a candidate reference gene another one is needed for its normalization. Several algorithms have been developed to deal with this problem. Vandesompele et al. introduced the use of the geometric mean of multiple reference genes in a normalization program named geNorm (Vandesompele et al., 2002). This application defines the gene stability as the average pairwise variation of a particular gene with all other control genes and ranks the genes according to their expression stability. An alternative program, NormFinder (Andersen et al., 2004), ranks the candidate reference genes based on the combined estimates of both intra- and intergroup variations.

Previous studies that investigated the effect of LXR agonist treatment on gene expression levels mostly made use of one of the traditional HKGs i.e. GAPDH, 18S or CycA (Abildayeva et al., 2006; Karten et al., 2006; Liang et al., 2004). Recently, suitable reference genes were described for rat brain tissue and hippocampal neurons under different experimental conditions (Bonefeld et al., 2008; Langnaese et al., 2008; Santos and Duarte, 2008). However, to our knowledge no study has yet been performed on glial cells. In the current study 10 candidate reference genes were selected and evaluated according to their expression stability in different rat glial cell cultures and in neonatal OLGs after LXR agonist treatment. 


\section{Materials and Methods}

\section{1) Cell culture}

Primary mature rat OLGs were isolated from whole brains of adult Wistar rats (Harlan, Horst, The Netherlands) as described by Yong and Antel (1992), with some minor modifications (Vanderlocht et al., 2006). Briefly, brain tissue was subjected to enzymatic dissociation with trypsin $(0.25 \%$, Gibco) and DNase I (100 $\mu \mathrm{g} / \mathrm{ml}$, Roche diagnostics, Vilvoorde, Belgium) and mechanical dissociation by passage through a $132 \mu \mathrm{m}$ mesh. Glial cells where separated from myelin debris and red blood cells by centrifugation on a $30 \%$ Percoll gradient. The mixed glial cell fraction was suspended in Dulbecco's modified Eagle medium (DMEM) (Gibco BRL, Paisley, UK) supplemented with 5\% FCS, penicillin (50 U/ml) and streptomycin (50 $\mu \mathrm{g} / \mathrm{ml})$. The cultures where selectively enriched for OLGs by means of differential adhesion to plastic. The non-adherent OLG fraction was plated at a density of $1.5 \times 10^{6}$ cells $/$ well on poly-L-lysine coated 24-well plates for qPCR. Cells were cultured for 11 days in DMEM supplemented with 5\% FCS, and subsequently for $72 \mathrm{~h}$ in SATO medium (Buttery and ffrench-Constant, 1999) with 0.5\% FCS. Four isolations of each six rats were tested.

Primary neonatal rat OLGs were isolated from newborn Wistar rats (postnatal day 1). Forebrains were collected, mechanically dissociated and the homogenate was cultured for 1012 days on Poly-L-lysine coated $(5 \mu \mathrm{g} / \mathrm{ml})$ cell culture flasks (Thermo Fisher Scientific/Nunc, Waltham, MA, USA) in DMEM (Gibco BRL) containing 10\% FCS, L-glutamine and penicillin/streptomycin (Gibco BRL). After a pre-shake to remove microglia (180 rpm, 1h), OLG progenitor cells, grown on top of a layer of astrocytes, were collected by shake-off ( 240 rpm, 20-24 h) as described (McCarthy and de Vellis, 1980) and cultivated for 2 days on polyL-lysine coated cell culture dishes in a defined SATO medium in presence of PDGF and FGF (Peprotech, Rocky Hill, NJ). OLG differentiation was induced by removing the growth factors 
and cultivating the cells for $72 \mathrm{~h}$ in SATO medium containing $0.5 \%$ FCS. Primary astrocytes were collected after OLG progenitor cells were shaken off, and used for further experiments. Primary neonatal rat OLGs were treated with either vehicle alone (ethanol) or $1 \mu \mathrm{M}$ LXR agonist T0901317 (Cayman Chemical, Tallinn, Estonia) for 72 hours. For the first experiment four preparations with an average of 10 rat pups per preparation were investigated, for the second experiment five preparations were used.

All animals were maintained in accordance with the Belgian and European animal welfare guidelines. The study was approved by the ethical committee for animal experiments of Hasselt University. The animals were housed under temperature controlled conditions, a $12 \mathrm{~h}$ light/dark cycle, and free access to food and water.

\section{2) RNA isolation and reverse transcription}

RNA was extracted from the cells using the RNeasy plus mini kit (Qiagen, Venlo, The Netherlands), according to the manufacturer's instructions. Genomic DNA was eliminated by a DNase-on-column treatment supplied with the kit. The RNA concentration was determined spectrophotometrically at $260 \mathrm{~nm}$ using the NanoDrop ND-1000 spectrophotometer (Thermo Fisher scientific, Waltham, USA) and RNA purity was checked by means of the absorbance ratio at $260 / 280 \mathrm{~nm}$. RNA integrity was assessed by electrophoresis on $2 \%$ agarose gels as indicated in the MIQE guidelines (Bustin et al., 2009). There was only a significant difference in RNA concentration between the mature oligodendrocytes and astrocytes but there were no significant differences between the other groups. Equal amounts of RNA input $(1 \mu \mathrm{g})$ were used in the subsequent cDNA synthesis reaction, which was performed using the Reverse Transcription System (Promega, Leiden, The Netherlands). Total RNA was incubated at $70^{\circ} \mathrm{C}$ for 10 min to prevent secondary structures. The RNA was placed on ice and supplemented 
with $\mathrm{MgCl}_{2}(25 \mathrm{mM})$, RTase buffer $(10 \mathrm{X})$, dNTP mixture $(10 \mathrm{mM})$, oligo d(t) primers, RNase inhibitor $(20 \mathrm{U})$ and AMV reverse transcriptase $(20 \mathrm{U} / \mu \mathrm{l})$. A tenfold dilution of the cDNA was made using nuclease-free water and stored at $-20^{\circ} \mathrm{C}$.

\section{3) Quantitative real time PCR}

qPCR was performed in an optical 96-well plate with an ABI PRISM 7500 fast sequence detection system (Applied Biosystems, Carlsbad, California) and universal cycling conditions $\left(10 \min 95^{\circ} \mathrm{C}, 40\right.$ cycles of $15 \mathrm{~s}$ at $95^{\circ} \mathrm{C}$ and $60 \mathrm{~s}$ at $\left.60^{\circ} \mathrm{C}\right)$. Each $10 \mu 1$ reaction contained $5 \mu \mathrm{l}$ SYBR Green Master Mix (Applied Biosystems), $0.3 \mu$ l gene-specific forward and reverse primers $(10 \mu \mathrm{M}), 2.5 \mu \mathrm{l}$ pre-diluted cDNA and $1.9 \mu 1$ nuclease-free water. No template controls contained nuclease-free water instead. Primer efficiency was determined by a standard curve of cDNA samples according to the MIQE guidelines for qPCR (Bustin et al., 2009). Amplification was followed by a melting curve analysis to check PCR product specificity. PCR products were also loaded on $2 \%$ agarose gels to confirm specificity of amplification and the absence of primer dimer formation. Data were analyzed using the $\Delta \Delta \mathrm{Ct}$ method (Livak and Schmittgen, 2001). Expression levels were normalized relative to the sample with the highest expression before geNorm (Vandesompele et al., 2002) v3.5 or NormFinder (Andersen et al., 2004) input. 


\section{Results}

Ten candidate reference genes were chosen from literature as well as from the geNorm website (HPRT, GAPDH, 18S, CycA, ActB, YWHAZ, Tbp, Rpl13A, HMBS, Pgk1). Full gene name, primer sequences, and amplicon length are shown in Table 1.

In the first part of our study, the stability of these candidate reference genes was tested in primary rat astrocytes and two primary rat OLG cultures. The OLGs were derived from either one day old or mature Wistar rats. The OLG cultures isolated from the adult rats are mature myelinating OLGs, which are differentiated further than the OLG cultures obtained from one day old rats. The latter are situated in the range of immature to non-myelinating mature OLGs (data not shown). geNorm was used to rank the tested genes according to their average expression stability measure ( $\mathrm{M}$ value) from the most stable (lowest $\mathrm{M}$ value) to the least stable (highest $M$ value). All studied genes except HMBS, have a high expression stability with $\mathrm{M}$ values below the default limit 1.5 (Vandesompele et al., 2002). geNorm identified CycA and Rpl13A as the most stable combination of reference genes, followed by Pgk1 (Fig 1A, Table 2). Next, the pairwise variation value, $V_{n / n+1}$, was analyzed. $V_{n / n+1}$ measures the stability/variation effect of including an additional reference gene on the normalization factor (that is calculated as the geometric mean of the selected reference genes), starting with genes with the $n$ lowest M-value. A small decrease in variation means that the added gene has no significant additional effect, and should not necessarily be included for calculation of a reliable normalization factor. The $\mathrm{V}_{\mathrm{n} / \mathrm{n}+1}$ graph (Fig 1B) shows that three reference genes are sufficient for normalization, since the inclusion of an additional reference gene increases the pairwise variation value. When normalising the genes of interest (cholesterol related genes) with an increasing number (3 to 8 ) of reference genes, no differences in expression levels were detected (data not shown). 
The same dataset was evaluated with the NormFinder software. This algorithm ranks the set of candidate genes according to their expression stability value in a given sample set and a given experimental design. YWHAZ, Pgk1 and Tbp were defined as the three most stable genes (Table 2). This ranking is not identical to the one determined by geNorm. However, both geNorm and NormFinder identified GAPDH, 18S and HMBS as most unstable genes. The NormFinder algorithm available in the GenEx Software also allows for the determination of the optimal number of control genes to be used in the normalization process, through the calculation of the Accumulated Standard Deviation. (Acc.SD). The Acc.SD of the ten reference genes is shown in figure 3A. The optimal number of reference genes is indicated by the lowest value for the Acc.SD. The lowest value, 0.3202, was found when five reference genes were used. However, the improvement of using five reference genes $(\mathrm{Acc} . \mathrm{SD}=0.3202)$ instead of only three $(\mathrm{Acc} . \mathrm{SD}=0.3284)$ is in this case very small. Therefore, the use of three genes is sufficient for good normalization.

In the second part of this study, the primary neonatal rat OLG culture was treated with the LXR agonist or with the vehicle (ethanol) alone. We opted for this culture since the cholesterol related genes had a higher expression level in the neonatal OLG culture compared to the mature OLG culture (data not shown). Here, geNorm identified CycA, Pgk1 and Rpl13A as the three most stable genes (Fig 2A, Table 3). These are the same genes as in the first part of this study but with a different ranking order. For the LXR agonist treatment of neonatal OLGs, the use of three reference genes for normalization is also sufficient, as the inclusion of an additional reference gene increases the $V_{n / n+1}$ value (Fig 2B) and normalization with more reference genes (3-6) gives the same levels of expression of the target genes (data not shown). 
For this data set, NormFinder identified the same genes as geNorm as most stable genes (Pgk1, CycA, Rpl13A) (Table 3). The Acc.SD reached its lowest value (0.1919), with three reference genes (Fig 3B).

To test the effect of different normalization factors on the expression levels of the gene of interest, one of the cholesterol related genes (Apolipoprotein E, ApoE) was normalised to the three most stable genes suggested by geNorm and Normfinder (CycA, Pgk1, Rpl13A). This result was compared with the result obtained after normalization with two of the commonly used "HKGs", GAPDH and 18S, which were identified as the most unstable genes by both algorithms. When the expression levels of the gene of interest after LXR agonist treatment were normalized with CycA, Pgk1 and Rpl13A, a significant upregulation of the gene compared to untreated cultures was found (Fig 4A). This effect disappears when GAPDH and 18S were used for normalization of ApoE mRNA expression levels (Fig 4B). A strong increase in standard error was detected in the control group after normalization with GAPDH and $18 \mathrm{~S}$ compared to the other normalization factor (Fig 4A and B). 


\section{Discussion}

Over the past several years, qPCR has become the method of choice for sensitive and accurate quantification of gene expression levels. Reliable quantification requires appropriate normalization, to correct for variation introduced by RNA quality and enzymatic efficiencies in PCR and reverse transcription. The most commonly used procedure to perform normalization is to relate the measured mRNA concentration for the gene of interest to the mRNA of a reference gene, that is expected to exhibit stable expression levels across various developmental and experimental conditions. Since no single gene is universally suitable for all purposes, these reference genes must be validated for each experimental setting to avoid misleading results (Dheda et al., 2004; Schmittgen and Zakrajsek, 2000; Vandesompele et al., 2002).

The current study is, to our knowledge, the first detailed description of the stability of rat reference genes in different glial cell types and after LXR agonist treatment in primary OLGs. The expression stability of ten commonly accepted candidate reference genes was analyzed. Two public programs, geNorm and NormFinder, were used for the evaluation of gene stability.

In a first study, the transcriptional stability was checked in neonatal and mature derived OLG cultures, and in a primary rat astrocyte culture. geNorm identified Rpl13A, CycA and Pgk1 as the most stable genes. The NormFinder output was not identical to the order of genes proposed by geNorm. This is not unexpected since both programs rely on different mathematical approaches (Andersen et al., 2004; Vandesompele et al., 2002). The pairwise

comparison approach, geNorm, tends to select those genes with the highest degree of similarity of expression profiles across the sample set. While NormFinder ranks the candidates with minimal estimated intra- and intergroup variation, and therefore selects the 
genes taking into account the different experimental conditions used. The top five genes in both algorithms, however, are comparable. What is remarkable is that both geNorm and NormFinder rank HMBS as well as the traditional reference genes GAPDH and $18 \mathrm{~S}$ as most unstable genes. Several other studies also described that GAPDH as well as other traditional reference genes are not suitable as internal reference genes (Dheda et al., 2004; Dheda et al., 2005). Therefore, we strongly advise to check the expression stability of these genes before using them for normalization purposes. Beside stability values, geNorm also calculates the pairwise variation value which determines the number of reference genes that should be used for normalization. Our data demonstrate that the use of the three most stable genes is sufficient for accurate normalization, since adding a fourth gene increases the pairwise variation value. The Acc.SD calculated by NormFinder indicated the use of five reference genes. However, since there are only minor differences between five $(\mathrm{Acc} . \mathrm{SD}=0.3202)$ and three $($ Acc. $\mathrm{SD}=0.3284)$ genes, three genes will be sufficient for normalization. When comparing the pairwise variation value and the Acc. SD., both geNorm and NormFinder show that the use of three reference genes is sufficient for good normalization and that addition of the most unstable gene, HMBS, increases the pairwise variation value and the Acc. SD.

In a second part of the study, the gene expression stability was examined in primary neonatal rat OLGs after LXR agonist treatment. The ranking of geNorm and NormFinder is identical. The most stable genes, CycA, Pgk1, Rpl13A, were also identified as most stable ones in untreated OLGs. In previous studies investigating total rat brain tissue under different experimental conditions, CycA, Pgk1 and Rpl13A were also identified as relatively stable genes (Bonefeld et al., 2008; Langnaese et al., 2008). These data suggest that the proposed genes are probably the most suitable reference genes for expression studies on rat brain or specific brain cells, although a careful screen for reliable reference genes is indispensable for each experimental design. For investigation of LXR agonist treatment in OLGs the use of 
three reference genes appears to be sufficient for appropriate normalization, since adding a fourth gene increases the pairwise variation value. The Acc. SD., identified by NormFinder, is lowest with 3 reference genes. Thus, both geNorm and NormFinder suggest using 3 reference genes for normalization. The Acc. SD. also increases when more than 6 genes are used, which is comparable to an increasing pairwise variation value when using 7 genes or more.

Different target gene expression results were obtained when performing normalization with the three best genes of geNorm and NormFinder (CycA, Pgk1, Rpl13A) compared to normalization with the commonly used HKGs GAPDH and $18 \mathrm{~S}$ which were defined as the most unstable genes. Similarly, when comparing normalization using ActB, with a normalization factor generated by geNorm, Bonefeld et al. demonstrated that in rat brain tissue the different approaches yield different qPCR results (Bonefeld et al., 2008). These findings again emphasize the importance of evaluating all genes used for normalization, even if they are traditionally used in other studies.

In conclusion, we evaluated ten candidate reference genes for normalization of gene expression levels in astrocytes and mature and neonatal OLGs and after LXR agonist treatment. Data suggest the use of a combination of CycA, Pgk1, Rpl13A or YWHAZ for normalization in the different cell cultures. During treatment of neonatal OLGs with the LXR agonist CycA, Pgk1 and Rpl13A can be used. Normalization with HMBS or the "traditional" housekeeping genes GAPDH and $18 \mathrm{~S}$ should be avoided in both experimental setups. Although these results could be an indication which reference genes could be used in glial cells, reference genes should always be evaluated for each individual experiment, since useful reference genes are very specific for every situation. 


\section{Acknowledgements}

KN was supported by a PhD grant from the tUL (transnational University Limburg). JJAH was supported by the FWO (Fonds Wetenschappelijk Onderzoek). The authors would like to thank Kelly Verstraete for the technical assistance. 


\section{References.}

Abildayeva K, Jansen PJ, Hirsch-Reinshagen V, Bloks VW, Bakker AH, Ramaekers FC, de Vente J, Groen AK, Wellington CL, Kuipers F, Mulder M. 24(S)-hydroxycholesterol participates in a liver X receptorcontrolled pathway in astrocytes that regulates apolipoprotein E-mediated cholesterol efflux. J Biol Chem, 2006; 281: 12799-808.

Andersen CL, Jensen JL, Orntoft TF. Normalization of real-time quantitative reverse transcription-PCR data: a model-based variance estimation approach to identify genes suited for normalization, applied to bladder and colon cancer data sets. Cancer research, 2004; 64: 5245-50.

Bonefeld BE, Elfving B, Wegener G. Reference genes for normalization: a study of rat brain tissue. Synapse (New York, N.Y, 2008; 62: 302-9.

Bustin SA, Benes V, Garson JA, Hellemans J, Huggett J, Kubista M, Mueller R, Nolan T, Pfaffl MW, Shipley GL, Vandesompele J, Wittwer CT. The MIQE guidelines: minimum information for publication of quantitative real-time PCR experiments. Clinical chemistry, 2009; 55: 611-22.

Buttery PC, ffrench-Constant C. Laminin-2/integrin interactions enhance myelin membrane formation by oligodendrocytes. Mol Cell Neurosci, 1999; 14: 199-212.

Deindl E, Boengler K, van Royen N, Schaper W. Differential expression of GAPDH and beta3-actin in growing collateral arteries. Molecular and cellular biochemistry, 2002; 236: 139-46.

Dheda K, Huggett JF, Bustin SA, Johnson MA, Rook G, Zumla A. Validation of housekeeping genes for normalizing RNA expression in real-time PCR. BioTechniques, 2004; 37: 112-4, 6, 8-9.

Dheda K, Huggett JF, Chang JS, Kim LU, Bustin SA, Johnson MA, Rook GA, Zumla A. The implications of using an inappropriate reference gene for real-time reverse transcription PCR data normalization. Analytical biochemistry, 2005; 344: 141-3.

Karten B, Campenot RB, Vance DE, Vance JE. Expression of ABCG1, but not ABCA1, correlates with cholesterol release by cerebellar astroglia. J Biol Chem, 2006; 281: 4049-57. 
Langnaese K, John R, Schweizer H, Ebmeyer U, Keilhoff G. Selection of reference genes for quantitative real-time PCR in a rat asphyxial cardiac arrest model. BMC molecular biology, 2008; 9:

53.

Liang Y, Lin S, Beyer TP, Zhang Y, Wu X, Bales KR, DeMattos RB, May PC, Li SD, Jiang XC, Eacho PI, Cao G, Paul SM. A liver $X$ receptor and retinoid $X$ receptor heterodimer mediates apolipoprotein $E$ expression, secretion and cholesterol homeostasis in astrocytes. J Neurochem, 2004; 88: 623-34. Livak KJ, Schmittgen TD. Analysis of relative gene expression data using real-time quantitative PCR and the 2(-Delta Delta C(T)) Method. Methods (San Diego, Calif, 2001; 25: 402-8.

McCarthy KD, de Vellis J. Preparation of separate astroglial and oligodendroglial cell cultures from rat cerebral tissue. J Cell Biol, 1980; 85: 890-902.

Peet DJ, Janowski BA, Mangelsdorf DJ. The LXRs: a new class of oxysterol receptors. Curr Opin Genet Dev, 1998; 8: 571-5.

Peinnequin A, Mouret C, Birot O, Alonso A, Mathieu J, Clarencon D, Agay D, Chancerelle Y, Multon E. Rat pro-inflammatory cytokine and cytokine related mRNA quantification by real-time polymerase chain reaction using SYBR green. BMC immunology, 2004; 5: 3.

Radonic A, Thulke S, Mackay IM, Landt O, Siegert W, Nitsche A. Guideline to reference gene selection for quantitative real-time PCR. Biochem Biophys Res Commun, 2004; 313: 856-62.

Repa JJ, Mangelsdorf DJ. The role of orphan nuclear receptors in the regulation of cholesterol homeostasis. Annu Rev Cell Dev Biol, 2000; 16: 459-81.

Saher G, Brugger B, Lappe-Siefke C, Mobius W, Tozawa R, Wehr MC, Wieland F, Ishibashi S, Nave KA. High cholesterol level is essential for myelin membrane growth. Nat Neurosci, 2005; 8: 468-75. Santos AR, Duarte CB. Validation of internal control genes for expression studies: effects of the neurotrophin BDNF on hippocampal neurons. J Neurosci Res, 2008; 86: 3684-92. 
Schmittgen TD, Zakrajsek BA. Effect of experimental treatment on housekeeping gene expression: validation by real-time, quantitative RT-PCR. Journal of biochemical and biophysical methods, 2000; 46: 69-81.

Schultz JR, Tu H, Luk A, Repa JJ, Medina JC, Li L, Schwendner S, Wang S, Thoolen M, Mangelsdorf DJ, Lustig KD, Shan B. Role of LXRs in control of lipogenesis. Genes Dev, 2000; 14: 2831-8.

Vanderlocht J, Hellings N, Hendriks JJ, Vandenabeele F, Moreels M, Buntinx M, Hoekstra D, Antel JP, Stinissen P. Leukemia inhibitory factor is produced by myelin-reactive T cells from multiple sclerosis patients and protects against tumor necrosis factor-alpha-induced oligodendrocyte apoptosis. J Neurosci Res, 2006; 83: 763-74.

Vandesompele J, De Preter K, Pattyn F, Poppe B, Van Roy N, De Paepe A, Speleman F. Accurate normalization of real-time quantitative RT-PCR data by geometric averaging of multiple internal control genes. Genome biology, 2002; 3: RESEARCH0034.

Vanmierlo T, Rutten K, Dederen J, Bloks VW, van Vark-van der Zee LC, Kuipers F, Kiliaan A, Blokland A, Sijbrands EJ, Steinbusch H, Prickaerts J, Lutjohann D, Mulder M. Liver X receptor activation restores memory in aged AD mice without reducing amyloid. Neurobiology of aging, 2009.

Wang L, Schuster GU, Hultenby K, Zhang Q, Andersson S, Gustafsson JA. Liver X receptors in the central nervous system: from lipid homeostasis to neuronal degeneration. Proc Natl Acad Sci U S A, 2002; 99: 13878-83. 


\section{Tables}

\section{Table 1 Reference genes selected for evaluation of expression stability}

\begin{tabular}{|c|c|c|c|c|c|c|}
\hline Symbol & name & forward and reverse primer & $\begin{array}{c}\text { Amplicon } \\
\text { size }\end{array}$ & $\begin{array}{c}\text { Primer } \\
\text { efficiency }\end{array}$ & $\begin{array}{c}\text { Cq value } \\
\text { (average } \pm \mathrm{SD})\end{array}$ & Reference* \\
\hline HPRT & Hypoxanthine phosphoribosyl-transferase & $\begin{array}{l}\text { CTCATGGACTGATTATGGACAGGAC } \\
\text { GCAGGTCAGCAAAGAACTTATAGCC }\end{array}$ & 123 & 93 & $23.23 \pm 1.52$ & (Peinnequin et al., 2004) \\
\hline GAPDH & Glyceraldehyde-3-phosphate dehydrogenase & $\begin{array}{l}\text { ACC ACA GTC CAT GCC ATC AC } \\
\text { TCC ACC ACC CTG TTG CTG TA }\end{array}$ & 452 & 100 & $24.22 \pm 3.36$ & * \\
\hline $18 \mathrm{~S}$ & $18 \mathrm{~S}$ subunit ribosomal RNA & $\begin{array}{l}\text { ACG GAC CAG AGC GAA AGC AT } \\
\text { TGT CAA TCC TGT CCG TGT CC }\end{array}$ & 310 & 107 & $21.05 \pm 2.86$ & Bonefeld et al., 2008 \\
\hline СусA & Cyclophilin A & $\begin{array}{l}\text { TAT CTG CAC TGC CAA GAC TGA GTG } \\
\text { CTT CTT GCT GGT CTT GCC ATT CC }\end{array}$ & 126 & 93 & $17.15 \pm 1.32$ & Langnaese et al., 2008 \\
\hline ActB & Beta actin & $\begin{array}{l}\text { TGT CAC CAA CTG GGA CGA TA } \\
\text { GGG GTG TTG AAG GTC TCA AA }\end{array}$ & 165 & 107 & $17.53 \pm 2.12$ & Bonefeld et al., 2008 \\
\hline YWHAZ & $\begin{array}{l}\text { Tyrosine 3-monooxygenase/tryptophan 5-monooxygenase activation } \\
\text { protein, zeta polypeptide }\end{array}$ & $\begin{array}{l}\text { GAT GAA GCC ATT GCT GAA CTT G } \\
\text { GTC TCC TTG GGT ATC CGA TGT C }\end{array}$ & 117 & 100 & $20.32 \pm 2.03$ & Langnaese et al., 2008 \\
\hline Tbp & TATA box binding protein & $\begin{array}{l}\text { TGG GAT TGT ACC ACA GCT CCA } \\
\text { CTC ATG ATG ACT GCA GCA AAC C }\end{array}$ & 131 & 93 & $26.33 \pm 2.62$ & Langnaese et al., 2008 \\
\hline Rpl13A & Ribosomal protein L13A & $\begin{array}{l}\text { GGA TCC CTC CAC CCT ATG ACA } \\
\text { CTG GTA CTT CCA CCC GAC CTC }\end{array}$ & 132 & 94 & $17.76 \pm 1.63$ & Langnaese et al., 2008 \\
\hline HMBS & Hydroxymethyl-bilane synthase & $\begin{array}{l}\text { TCC TGG CTT TAC CAT TGG AG } \\
\text { TGA ATT CCA GGT GAG GGA AC }\end{array}$ & 176 & 99 & $24.62 \pm 2.49$ & Bonefeld et al., 2008 \\
\hline Pgk1 & Phosphoglycerate kinase 1 & $\begin{array}{l}\text { ATG CAA AGA CTG GCC AAG CTA C } \\
\text { AGC CAC AGC CTC AGC ATA TTT C }\end{array}$ & 104 & 97 & $21.07 \pm 1.58$ & Langnaese et al., 2008 \\
\hline ApoE & Apolipoprotein E & $\begin{array}{l}\text { CCT GAA CCG CTT CTG GGA TT } \\
\text { GCT CTT CCT GGA CCT GGT CA } \\
\end{array}$ & 65 & 107 & $16.96 \pm 1.68$ & (Vanmierlo et al., 2009) \\
\hline
\end{tabular}

* Primers were designed by our laboratory using Oligo6 software. 
Table 2 Candidate reference genes for normalization in different glial cell cultures ranked according to their expression stability by geNorm and NormFinder

\begin{tabular}{|c|c|c|c|c|c|}
\hline \multicolumn{3}{|c|}{ geNorm } & \multicolumn{3}{c|}{ NormFinder } \\
\hline \multirow{2}{*}{ Ranking order } & Gene name & Average M value & Ranking order & Gene name & Stability value \\
\hline 1 & Rpl13A & 0.356 & 1 & YWHAZ & 0.34 \\
1 & CycA & 0.356 & 2 & Pgk1 & 0.376 \\
3 & Pgk1 & 0.506 & 3 & Tbp & 0.445 \\
4 & YWHAZ & 0.733 & 4 & ActB & 0.507 \\
5 & Tbp & 0.921 & 5 & CycA & 0.539 \\
6 & HPRT & 1.059 & 6 & Rpl13A & 0.559 \\
7 & ActB & 1.168 & 7 & HPRT & 0.627 \\
8 & GAPDH & 1.252 & 8 & GAPDH & 0.7 \\
9 & 18S & 1.356 & 9 & $18 \mathrm{~S}$ & 0.864 \\
10 & HMBS & 1.652 & 10 & HMBS & 0.919 \\
\hline
\end{tabular}

Table 3 Candidate reference genes for normalization in primary neonatal rat OLG during LXR agonist treatment ranked according to their expression stability by geNorm and NormFinder

\begin{tabular}{|c|c|c|c|c|c|}
\hline \multicolumn{3}{|c|}{ geNorm } & \multicolumn{3}{|c|}{ NormFinder } \\
\hline \multirow{2}{*}{ Ranking order } & Gene name & Average M value & Ranking order & Gene name & Stability value \\
\hline 1 & Pgk1 & 0.535 & 1 & Pgk1 & 0.294 \\
1 & CycA & 0.535 & 2 & CycA & 0.338 \\
3 & Rpl13A & 0.579 & 3 & Rpl13A & 0.341 \\
4 & HPRT & 0.689 & 4 & HPRT & 0.419 \\
5 & YWHAZ & 0.898 & 5 & YWHAZ & 0.556 \\
6 & ActB & 0.974 & 6 & ActB & 0.676 \\
7 & Tbp & 1.346 & 7 & Tbp & 0.741 \\
8 & $18 S$ & 1.705 & 8 & $18 S$ & 0.946 \\
9 & GAPDH & 2.045 & 9 & GAPDH & 1.265 \\
\hline
\end{tabular}




\section{Figure legends}

Figure 1 Evaluation of reference genes using geNorm in different glial cell cultures.

(A) Average expression stability measure (M) of 10 reference genes, during stepwise exclusion of the least stable control gene. Lower $\mathrm{M}$ value of average expression stability indicates more stable expression. (B) Determination of the optimal number of control genes for normalization on the basis of a pair-wise variation $\left(\mathrm{V}_{\mathrm{n} / \mathrm{n}+1}\right)$ analysis. Every bar represents the change in normalization accuracy when stepwise adding more endogenous controls according to ranking in fig $1 \mathrm{~A}$.

Figure 2 Evaluation of reference genes using geNorm in primary neonatal rat OLGs during LXR agonist treatment.

(A) Average expression stability measure (M) of 10 reference genes, during stepwise exclusion of the least stable control gene. Lower $M$ value of average expression stability indicates more stable expression. (B) Determination of the optimal number of control genes for normalization on the basis of a pair-wise variation $\left(\mathrm{V}_{\mathrm{n} / \mathrm{n}+1}\right)$ analysis. Every bar represents change in normalization accuracy when stepwise adding more endogenous controls according to ranking in fig $2 \mathrm{~A}$.

Figure 3 Determination of the optimal number of reference genes for normalization based on the calculation of the Acc. SD. (A) in different glial cell cultures and (B) during LXR agonist treatment. The lowest value for the Acc. SD. in panel A was found when 5 reference genes were used, in panel B when 3 reference genes were used. Data analysis was performed by NormFinder. 
Figure 4 qPCR data vary depending on choice of reference genes.

The cholesterol related gene was normalized with the geometric mean (GM) of the three best genes of geNorm and NormFinder (CycA, Pgk1, Rpl13A) (A) and the GM of the most unstable genes GAPDH and $18 \mathrm{~S}$ according to both programs (B). Results are presented as mean \pm SEM of five independent preparations $(n=5)$. Statistics: Kruskall Wallis test (post test: Dunns, compares all pairs of columns). $\mathrm{P} \leq 0.05$ is considered statistically significant. 